\title{
Work-related Musculoskeletal Disorders among mail sorting officers in Sri Lanka: a cross sectional study
}

\author{
S.S.P. Warnakulasuriya ${ }^{1}$, R.J. Peiris-John ${ }^{2}$, S. Sivayogan ${ }^{3}$, N. Sathiakumar ${ }^{4}$, D. Coggan ${ }^{5}$, A.R. Wickremasinghe ${ }^{6}$
}

\begin{abstract}
Background: Mail sorting requires repetitive movements of the upper limbs, target oriented tasks, long term standing or sitting, concentration and a good memory. The extent of musculoskeletal disorders (MSDs) among mail sorting officers (MSOs) is not known. This study was conducted to determine the prevalence and associated factors with work-related MSDs among MSOs in Sri Lanka.
\end{abstract}

Methods: 250 randomly selected MSOs who were attached to the Central Mail Exchange, Colombo, were administered a pre-tested questionnaire after obtaining informed written consent. Information on demographic characteristics, musculoskeletal symptoms of the different body parts, job tasks, work history, job satisfaction, sick leave, feelings of job security, and personal perceptions of musculoskeletal symptoms during the past month were obtained. Data were analyzed using SPSS (version 16). Descriptive analyses were used to describe variables and chi-square tests were used to determine associations between pain symptoms and associated variables.

Results: The prevalence of backache was highest (38.4\%), followed by shoulder pain (27.6\%), knee pain (21.2\%), neck pain (16.4\%), wrist and arm pain (11.2\%) and elbow pain (10\%). 59.6\% of MSOs had experienced at least one MSDs sometime during the past 12 months. Mail sorting activities involved repetitive hand and wrist movements for more than four hours a day $(n=239)$, repetitive bending and straightening of elbow more than one hour a day $(n=239)$, keeping the hand above shoulder height for more than one hour a day $(n=239)$ and climbing up and down more than 30 steps $(n=218)$ a day. Height was significantly associated with shoulder pain $(\mathrm{OR}=1.056,95 \% \mathrm{CI}=1.069-3.577)$. Pain in all of the other sites was not associated with known risk factors in other studies.

Conclusion: The predominant ergonomic problem in mail sorters in Sri Lanka are in the lower back, shoulder and neck areas. Although there is a high prevalence of some musculoskeletal symptoms in MSOs, most of the known risk factors identified in other studies such as age, right or left handedness, years of experience in current job, smoking habit and job satisfaction etc., were not significantly associated with MSDs in this population. Other physical, psychosocial and environmental factors need to be investigated.

\section{Introduction}

Musculoskeletal disorders of the back, neck and upper limb are a major cause of morbidity and disability with a substantial economic impact. In some cases, the symptoms arise from identifiable pathology in the spine or arm such as a herniated inter-vertebral disc or nerve-root compression in the carpal tunnel. Most often, however, the underlying pathology is unclear, and the symptoms are classified as "non-specific".

Epidemiological research has linked the occurrence of back, neck and upper limb disorders with various physical activities in the workplace (1) and with psycho-social influences such as low mood and job dissatisfaction (2). However, these established risk factors do not adequately explain the striking temporal changes that have been observed in disability attributed to such disorders. For example, in Britain, rates of incapacity for work because of back problems increased more than seven-fold between 1953 and 1992 at a time when the physical demands of work were generally reducing (3) .In Australia, too, a major epidemic of disability from arm pain was reported in the early 1980 s that was not found in other countries where similar technologies and working methods were employed (4). This has prompted the hypothesis that the occurrence and chronicity of non-specific musculoskeletal complaints and resultant disability are importantly determined by cultural influences and health beliefs as well as by physical activities and mental health status (5). Within populations that are culturally predisposed to chronic and disabling non-specific

\footnotetext{
1. Department of Medical Education and Health Sciences, Faculty of Medical Sciences, University of Sri Jayewardenepura.

Correspondence: ashara96@yahoo.com

2. Section of Epidemiology and Biostatistics, School of Population Health, Faculty of Medical and Health Sciences, University of Auckland.

3. Department of Community Medicine, Faculty of Medical Sciences, University of Sri Jayewardenepura.

4. Departments of Epidemiology, School of Public Health, University of Alabama at Birmingham, USA.

5. Epidemiology Resource Center, Southampton General Hospital, Southampton, United Kingdom.

6. Department of Public Health, Faculty of Medicine, University of Kelaniya, Sri Lanka.
} 
musculoskeletal illness, individual risk is influenced by a general tendency to report and worry about common somatic symptoms. This "somatising tendency" has been found to be associated with the prevalence of musculoskeletal symptoms and medical consultation for such symptoms (6). "Somatising" tendency also predict the incidence of musculoskeletal symptoms and their persistence once they have developed (7). However, there is a need for more research to extend these observations to other cultural settings.

The main objective of occupational health of a country is to ensure that every employee has a safe and healthy work environment in order to preserve human recourses (8). Most of the developed countries have achieved this objective. However, many developing countries such as Sri Lanka have yet to achieve these targets. Some of the major reasons for the delay of having adequate occupational health interventions are the lack of priority given to occupational health as a whole, lack of sound policies and, in particular, the lack of scientific studies on occupational health and safety that may inform policy in these settings. The aim of this study was to determine the prevalence and associated risk factors of work-related musculoskeletal symptoms among mail sorting officers (MSOs) in Sri Lanka.

\section{Methods}

250 MSOs were randomly selected from 480 MSOs working in the Central Mail Exchange in Colombo. Given that there were only 480 MSOs in the Central Mail Exchange, a sample of $218 \mathrm{MSOs}$ was required to estimate a prevalence of $50 \%$ with an alpha error of 0.05 and a confidence interval of $10 \%$ (Lwanga and Lemeshow 1991). Assuming that $15 \%$ of the sample may decline to participate, $250 \mathrm{MSOs}$ were selected, all of whom agreed to participate.

Participants were from different sections of the mail handling department and worked on a pre-scheduled rotational basis in each of these sections. There was no difference in the working conditions in each section.

A questionnaire was developed in English and translated into Sinhala, the main language spoken by the majority of the population. The Sinhala version was back translated into English by an independent person and the two English versions were found to be comparable. The Sinhala version of the questionnaire was pre-tested on ten MSOs in other post offices in Colombo.

The pre-tested questionnaire was administered to the MSOs from August 2009 to October 2009 in face to face personal interviews. Interviews were conducted at the work place maintaining privacy and confidentiality. Information on demographic characteristics, musculoskeletal symptoms in different anatomical locations, job tasks, work history, job satisfaction, sick leave, job security and personal perceptions of musculoskeletal symptoms and mood during the past month were obtained. A body "map" was used to assist workers to pinpoint body sites at which symptoms occurred. The duration of pain, whether treatment was taken and the workers expectations on the recurrence of pain in the same body regions in future were also obtained. Workers were requested to comment on their beliefs regarding musculoskeletal pain in relation to work, rest and reduction of pain. Workers rated their mood during the past month on a rating scale ranging from happiness at all times to no happiness at all or depression. Though all participants could understand English, all the interviews were conducted in Sinhala, the common language spoken by all study population including few Tamils and Moors working in the central mail exchange .

Data gathered from the structured interviews were analyzed using the SPSS software package (version 13).Descriptive analysis including frequencies, percentages and univariate analysis for continuous variables were performed. Chi-square tests were used to identify the significant associations between risk factors and self reported musculoskeletal symptoms. Odds ratios and their $95 \%$ confidence intervals were calculated for each independent variable.

Ethical clearance for the study was obtained from the Ethical Review Committee of the Faculty of Medical Sciences, University of Sri Jayewardenepura. Informed written consent was obtained from each person prior to participation. Written permission was obtained from the Post Master General, Ministry of Posts and Telecommunications prior to conducting the survey.

\section{Results}

All participants were males (no female MSOs in Sri Lanka). Most (94\%) were between ages of 39 to 59 years of age (Table 1). The majority of them was Sinhalese $(96.9 \%)$ and $93.8 \%$ of MSOs were right handed.

$82 \%$ of the participants had over five years of work experience. Heights of MSOs ranged from $143 \mathrm{~cm}$ to $189 \mathrm{~cm}$ with a mean $( \pm \mathrm{SD})$ height of $165.31( \pm 7.45)$ $\mathrm{cm}$. Though various concerns regarding the job were reported by the participants during the interview, the majority of them was either very satisfied (47.2\%) or satisfied $(52.2 \%)$ with their current job. There were $49.4 \%$ of the workers who regularly smoked in the past and $23.5 \%$ still continue to smoke. Sick leave 
taken by MSOs due to musculoskeletal disorders during the past year was low; $6 \%$ of them had taken 1-5 days leave, $3.3 \%$ had taken 6-30 days leave and $0.8 \%$ had taken more than 30 days leave.
Reported mail sorting activities involved repetitive hand and wrist movements for more than 4 hours a day $(n=239)$, repetitive bending and straightening of the elbow more than one hour a day $(n=239)$, keeping

Table 1: Characteristics of the participants

\begin{tabular}{|c|c|c|}
\hline Characteristics & $\begin{array}{c}\text { Number of Subjects } \\
n=250\end{array}$ & $\%$ \\
\hline \multicolumn{3}{|l|}{ Age } \\
\hline $20-29$ & 01 & 0.4 \\
\hline $29-39$ & 14 & 5.5 \\
\hline $39-49$ & 110 & 44.2 \\
\hline $49-59$ & 125 & 49.9 \\
\hline \multicolumn{3}{|l|}{ Ethnicity } \\
\hline Sinhalese & 242 & $96 . .9$ \\
\hline Tamil & 01 & 0.4 \\
\hline Moor & 07 & 2.7 \\
\hline \multicolumn{3}{|l|}{ Handedness } \\
\hline Right & 234 & 93.8 \\
\hline Left & 16 & 6.2 \\
\hline \multicolumn{3}{|c|}{ Years of $W$ ork experience } \\
\hline $1-5$ years & 46 & 18 \\
\hline$>5$ years & 204 & 82 \\
\hline \multicolumn{3}{|l|}{ Height } \\
\hline$<160 \mathrm{~cm}(\mathrm{n}=67)$ & 67 & 26.8 \\
\hline$>=160 \mathrm{~cm}(\mathrm{n}=183)$ & 183 & 73.2 \\
\hline \multicolumn{3}{|l|}{ Job satisfaction } \\
\hline Very satisfied & 109 & 43.7 \\
\hline Satisfied & 134 & 53.5 \\
\hline Unsatisfied & 6 & 2.4 \\
\hline Very unsatisfied & 1 & 0.4 \\
\hline \multicolumn{3}{|l|}{ Smoking habit } \\
\hline Ever smoked & 186 & 72.9 \\
\hline Current smoker & 60 & 23.5 \\
\hline Non smoker & 64 & 27.1 \\
\hline \multicolumn{3}{|l|}{ Sick leave taken } \\
\hline \multicolumn{3}{|c|}{ for musculoskeletal disorders } \\
\hline 1-5 days & 15 & 5.9 \\
\hline 6-30days & 8 & 3.3 \\
\hline$>30$ days & 2 & 0.8 \\
\hline
\end{tabular}

During the preceding 12 months, back pain was prevalent in $38.4 \%(\mathrm{n}=96)$ followed by shoulder pain in $27.1 \%(\mathrm{n}=67)$, knee pain in $21.2 \%(\mathrm{n}=52)$, neck pain in $16.4 \%(\mathrm{n}=42)$, wrist and arm pain in $11.2 \%(\mathrm{n}=28)$ and elbow pain in $10.4 \%(\mathrm{n}=29)$. Of the people with back pain, $10.4 \%$ reported symptoms that meet the diagnostic criteria for sciatica; $19.6 \%$ sought treatment from a doctor. the hand above shoulder height for more than one hour a day $(\mathrm{n}=239)$ and climbing up and down more than 30 steps a day $(n=218)$. The number of working hours per week ranged from 48 to 96 hours (mean= 63 hours, $\mathrm{SD}=11.45$ ). Thirty two percent of the participants worked under pressure to complete the given tasks.. $11.6 \%$ of those with knee pain had sought medical treatment. Very few people with neck 
pain $(5.6 \%)$ and shoulder pain $(9.6 \%)$ had sought medical treatment while the others had used home remedies for pain symptoms. The majority of the participants $(82 \%)$ knew that repetitive body movements cause musculoskeletal symptoms.

The association between identified risk factors and pain in the back, neck, and knee and shoulder, and elbow, and wrist and hand are given in Tables 2 and 3 , respectively. Height of MSOs was significantly associated with shoulder pain; the prevalence of shoulder pain was higher in short persons as compared to tall persons.

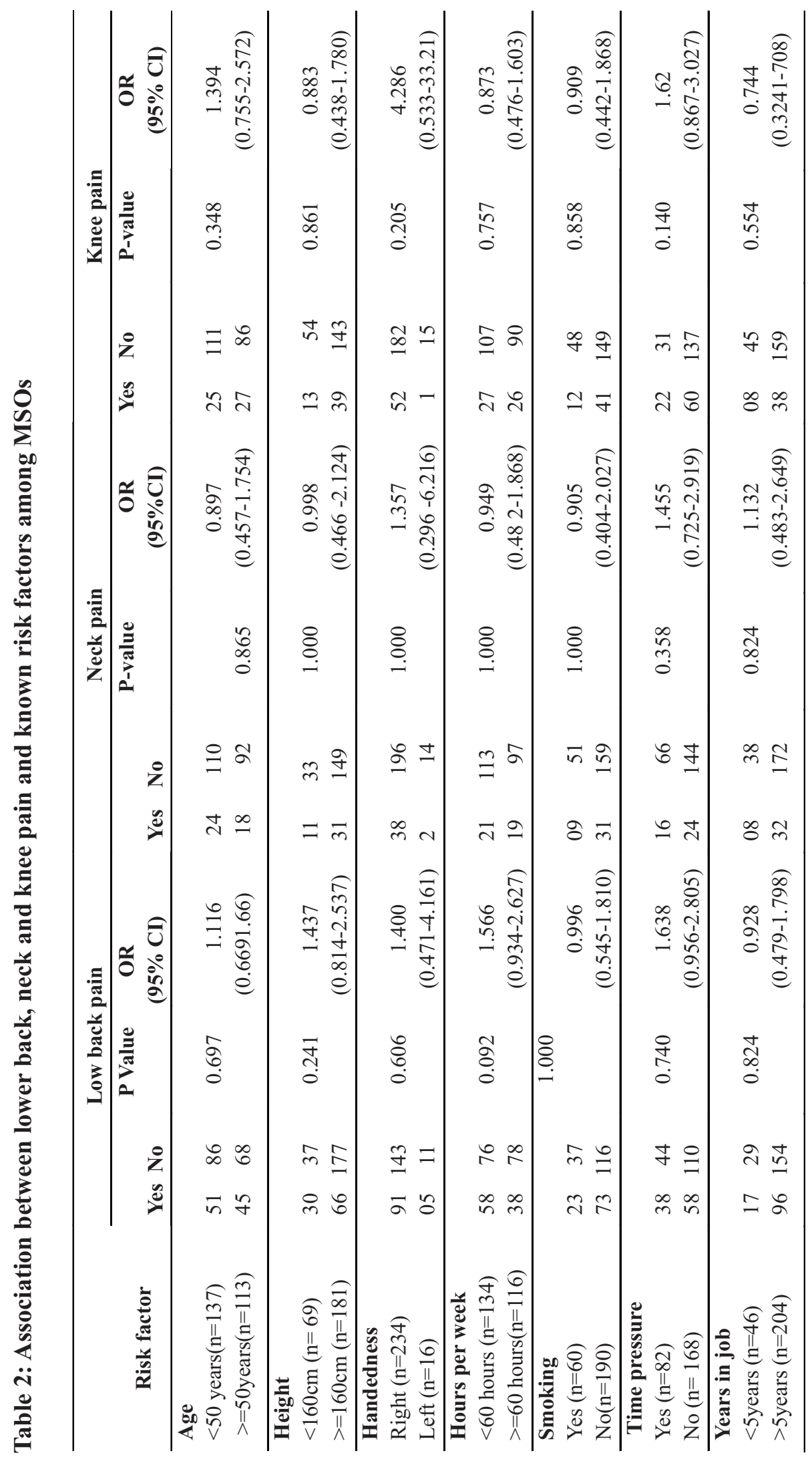




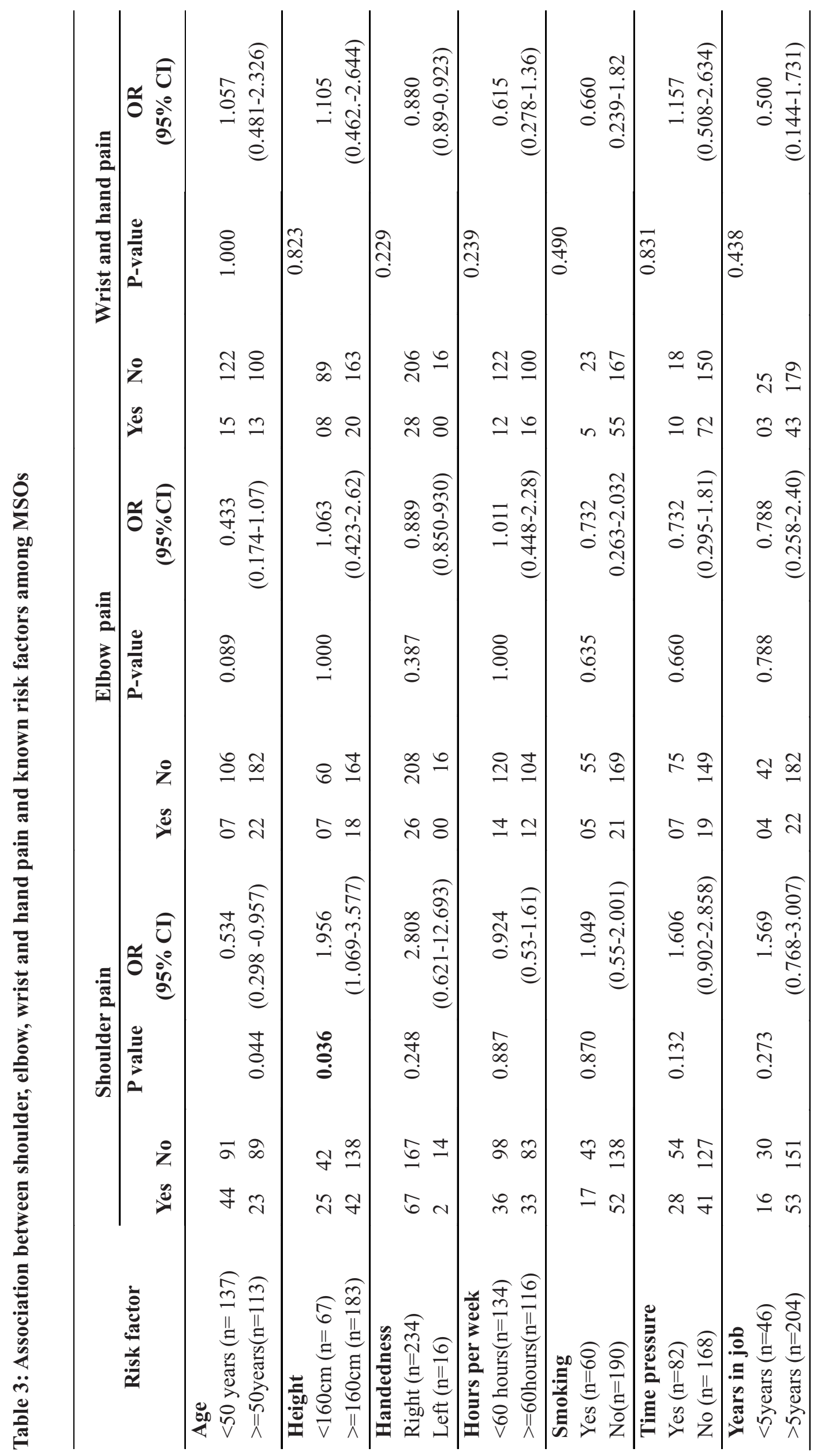




\section{Discussion}

The prevalence of musculoskeletal symptoms such as pain in the back $(38.4 \%, 95 \%$ CI $=0.3252$ $0.4455)$, shoulder $(27.6 \%, 95 \% \mathrm{CI}=0.2233$ $0.3339)$, knee $(21.2 \%, 95 \% \mathrm{CI}=0.1647-0.2660)$, neck $(16.4 \%, 95 \% \mathrm{CI}=0.1219-0.2138)$, wrist and hand $(11.2 \%, 95 \% \mathrm{CI}=0.0772-0.1557)$, and elbow $(10.4 \%, 95 \% \mathrm{CI}=0.0705-0.1466)$ is high among MSOs at the Central Mail exchange in Colombo, Sri Lanka. Low back pain was the commonest musculoskeletal symptom reported.

Mail sorters need to be able to concentrate, have a good memory and reading skills, and work with speed and accuracy in checking names, addresses and numbers. In Sri Lanka, the Postal Department recruits MSOs only from among experienced postmen and porters who have at least 11 years of schooling. Additionally, they must be competent in reading and writing in the three official languages used in the country (Sinhala, Tamil and English), be physically fit and be able to sort mail proficiently. One MSO is expected to sort at least 7000-8000 light letters during an eight hour duty shift.

The current study was confined to the Central Mail Exchange situated in Colombo, the commercial capital of Sri Lanka. MSOs at the Central Mail Exchange work round the clock on shift duty and very often do double shifts (up to 16 hours per day). During working hours these workers have repetitive neck, shoulder, elbow and wrist movements while having to maintain their concentration to sort mail by names and addresses. Further, they work long hours sitting on stools with no back support or keep standing with their hands held above shoulder level for a considerable period of time. In addition, the target oriented tasks, time pressure and repetitive movements of the body parts in the process of mail sorting often tends to increase the level of stress among MSOs. The physical and psychosocial strains associated with the very nature of their occupation, makes MSOs at risk of developing musculoskeletal symptoms. This is the first report on work-related musculoskeletal disorders among MSOs in Sri Lanka.

Musculoskeletal disorders of the back, neck and upper limb are a major cause of morbidity and disability with a substantial economic impact $(9,10)$. A study conducted in New Zealand among postal workers found a high prevalence of elbow and wrist/ hand pain (13). Manual letter sorting is a mildly repetitive job with the pace of work being controlled by the worker. Mail sorting, being a target oriented job, may lead to increased stress. Stress is a known risk factor of musculoskeletal disorders $(14,15,16)$. Some findings of this study are different to those reported from studies on people involved in various other manual tasks $(11,17,18)$
Several studies have shown individual factors such as age, height, right or left handedness, years of experience in current job, smoking habit, job satisfaction and mental stress are associated with musculoskeletal disorders $(9,18,19)$. In this study, only height was associated with shoulder pain. MSOs shorter than $160 \mathrm{~cm}$ had a significantly higher prevalence of shoulder pain probably because shorter MSOs need to exert more to reach the upper shelves of mail sorting racks than taller MSOs.

Repetitive movements of the shoulder, the elbow and the wrist were not associated with musculoskeletal disorders among MSOs in Sri Lanka. Repetitive movements have been shown to be associated with musculoskeletal disorders in different body regions in some studies (20). It is likely that this may be due to cultural influences. According to the available evidence, cultural influences play a major role in the development of musculoskeletal disorders (21). Cultural factors such as health beliefs and expectations have an important influence on the occurrence of musculoskeletal disorders and associated disability (11).

There is accumulating evidence of psychosocial factors and increased work demands being important predictors of musculoskeletal disorders (22, 23).Although time pressure was not significantly associated with musculoskeletal disorders in our study, Hoffmann, E.R., Macdonald, W.A. and Almond, G.C suggest that sorting mail can impose a high cognitive demand due to processing of a large amount of information under time pressure (24). Insufficient time for the performance of such tasks increases stress.

Some psychological task factors, such as increased time working under deadlines, increased job pressure, and the lack of participation in decision making, promote the development of musculoskeletal disorders because they tend to increase stress levels in people (25). Physiological correlates of 'stress' can directly damage body tissues in ways that increase the risk of musculoskeletal disorders in different occupational groups. Tasks that require a substantial amount of cognitive 'information processing', to a degree that slows down the rate at which it is possible to work effectively, constitute a cognitive hazard. Such tasks include those that require things to be sorted or categorized such as mail sorting, product inspection, etc (9).

Some studies report a positive association between musculoskeletal disorders and low self control, poor social support by colleagues and a combination of these among postal workers (14.18). No such associations were observed in this study. 
Bjorksten, M.G., Edling,2001 found high mental pressure to be a significant risk factor in the development of musculoskeletal disorders of the neck and shoulder. The high level of job satisfaction among the Sri Lankan MSOs indicates that mental stress may not be a problem. This may be a reason for the absence of an association between stress and musculoskeletal disorders in this population despite the number of working hours per week being considerably high. Moreover, in spite of experiencing musculoskeletal symptoms, their reluctance to absent themselves from work indicates their willingness and motivation to work and their preference for not being labeled as sick people. Other reasons may include financial compulsion to work as they receive a comparatively low salary. Personal beliefs about MSDs and other social needs may be preventing them from getting absent from work and from seeking treatment from a medical practitioner. It is unlikely that the healthy worker effect would have influenced our results as $80 \%$ of the workers had more than 5 years experience in the current job. This would have provided sufficient time for musculoskeletal disorders to develop in this population.

Although a number of possible risk factors that contribute to musculoskeletal disorders in MSOs have been documented, in this study, we only found a significant association between height and the shoulder pain. It is likely that there are other physical, psychosocial and environmental factors that may be contributing to musculoskeletal disorders that were not addressed by the study instrument used in this study. Tinubu et al. explained the reasons for the variation of results in different studies on musculoskeletal disorders as subjectivity of terms, variations in instruments, organizational differences in work settings, and cultural differences in the perception and reporting of pain and disorders (21). Further, factors such as physical load, organizational factors (physical demand at work, unrewarding work), individual characteristics and psychosocial factors that were not addressed in the questionnaire have been found to be related to various musculoskeletal symptoms $(26,21)$. For example, a history of the illness, prior injuries, weight and obesity, recurrent night duty, and long distance to work place may be some such factors that influence the occurrence of musculoskeletal disorders in this population. The high response rate in the current study $(100 \%)$ suggests that there is a high level of interest with respect to work-related musculoskeletal disorders that exist among MSOs investigated in this study.

The working environment, conditions of work and the load of mail sorting work in other regional and private sector post offices in Sri Lanka is likely to differ from that of the Central Mail Exchange. Hence, the generalizability of our results to these subgroups is limited.

\section{Conclusion}

The predominant musculoskeletal disorder in mail sorters in Sri Lanka is pain in the lower back, neck and shoulder area. Only height was associated with shoulder pain. None of the other known risk factors found in other studies were not found significantly associated with musculoskeletal symptoms in present study. A long-term longitudinal cohort study that assesses all the physical, psychosocial, environmental, individual and cultural factors that increase the risk of musculoskeletal disorders will help in studying the problem in greater depth, so that interventions may be designed to reduce the prevalence of musculoskeletal disorders among this occupational group.

\section{Acknowledgement}

This study was funded by the International Training and Research in Environmental and Occupational Health (ITREOH) Program of the University of Alabama at Birmingham (National Institutes of Health and the Fogarty International Center : Grant number 5 D43 TWO5750).

\section{References}

1. US Department of Health and Human Sciences, 1997

2. Bongers PM, DeWinter CR, Kompier MAJ, Hildebrandt VH. Psychosocial factors at work and musculoskeletal disease. Scand J Work Environ Health 1993; 19:297-312.

3. Clinical Stranded Advisory Group. Epidemiological Review. The epidemiology and cost of low back pain.HMSO.1994

4. Gun RT. The incidence and distribution of RSI in South Australia 1980-81 to 1986-87. Med J Austral 1990; 153:376-380.

5. Coggon D. Occupational Medicine at a turning point. Occupational and Environmental Medicine 2005; 62:281-283

6. K. T. Palmer, C. Cooper, K. Walker-Bone, H. Syddall and D. Coggon Use of keyboards and symptoms in the neck and arm: evidence from a national survey .Occupational medicine..2003:51(6):392-395

7. Ryall, C., Coggon D.,, Peveler R., Poole J.T., Palmer K.T., : A prospective study of arm pain in primary care and physiotherapy: prognostic determinants. Rheumatology .2007:46:508-515.

8. Parimalan, P.Kamalamma, N.Ganguli, A.K.:.Knowlade, attitude and Practices Related to Occupational Health problems among Garment workers in Taminadu India.Jounal of Occupational Health. 2007, 49:528-534

9. Australian Safety and Compensation Council: Work Related Musculoskeletal Diseases in Australia. 2006. Retrieved from www.nohsc.gov.au on 02.11.2010

10. Cock,M.N., Pirotte, K.C. Dombrecht, B.J.Organizational and Psychosocial factors and 
development of musculoskeletal Disorders.Final report,2004, 1-6

11. Madan, I., Reading,I., Palmer, K.T.,Coggon, D.:Cultural Diffrence in Musculoskeletal Symptoms and Disability. The International Journal of Epidemiology, 2008,1-9

12. Lawren H. Daltroy, .P.H., Maura D. Iversen, B.S.P.T., Martin G. Larson, S.D., Robert Lew, Elizabeth Wright., Ryan.J, Zwerling, C. Fossel,A.H. and Matthew H. Liang, . A Controlled Trial of an Educational Program to Prevent Low Back Injuries. 1997, 337:322-328 Harcombe H

13. , McBride D, Derrett S, Gray A.: Physical and psychosocial risk factors for musculoskeletal disorders in New Zealand nurses, postal workers and office workers. Department of Preventive and Social Medicine, University of Otago, Dunedin, New Zealand,2010.

14. Wahlstedt, K.G.I., Bjorksten, M.G., Edling, C.2001.: International Journal of Behavioral Mediicine.8 (3), 2003, 181-103.

15. Smedley,J. Inskip, H. Trevelyan,H. Buckle,P. Cooper, C.Coggon,D. Risk factors for incident neck and shoulder pain in hospital nurses. Occupational and Environvironmental medicine 2003;60:864-869

16. Lei, L, Liang,Y. Prevalence and risk factors of musculoskeletal disorders of foundry workers .Department of Occupational Health, FUDAN University School of Public Health. (2004)

17. Campo. M. Weiser, S.Koenig, K.L.,Nordin,M. WorkRelated Musculoskeletal Disorders in Physical Therapists Prospective Cohort study 1-year Followup. 2007

18. Tiwari, R.R.,Pathak,M.C.,Zodpey,S. P.Low back Pain among Textile workers. Indian Journal of Occupational and Environmental Medicine. 2003, Vol.7.No1

19. Brandt L.P.A, Andersen J.H, Lassen C.F, Kryger A, Overgaard E, Vilstrup I, Mikkelsen.S. Neck and shoulder symptoms and disorders among Danish computer workers. Scand J Work Environ Health 2004; 30 (5):399-409.

20. Varmazyar,S., varyani,A.S., Zeidi,I.M., Hashemi,H.J.:,Evaluation Working Posture and Musculoskeletal Disorders Prevalence in Pharmacy Packaging Workers. European Journal of Scientific Research.2009.29(1): :82-88

21. Tinubu M.S ,Chidozie E Mbada,Adewale L Oyeyemi Ayodele A Fabunmi :Work-Related Musculoskeletal Disorders among Nurses in Ibadan, South-west, Nigeria: a cross-sectional survey. $B M C$ Musculoskeletal Disorders , 2010, 11:12

22. Skove,T ,Borg ,V and E Orhede :Psychosocial and Physical Risk Factors for MusculoSkeletal Disorders of Neck Shoulder and Lower back among sales people. Occupational and Environ Medicine 1996, 53:351-356

23. Josephson, M.: Work factors and musculoskeletal disorders - An Epidemiological approach focusing on female nursing personnel. 1998

24. Hoffmann, E.R., Macdonald, W.A. and Almond, G.C. Quantification of the cognitive difficulty of mail sorting. International Journal of Industrial Ergonomics, .1993. 11,83-98
25. Sauter, B.B.S. Petersen,L.F.M.. Psychosocial risk factors for work-related Musculoskeletal disorders among Newspaper employees. Thomas Hales Scand JWork Environ Health. 1994, 20:417-26

26. Mencken, H.L. Risk Factors for Musculoskeletal Disorders: "There is always an easy solution to every human problem - neat, plausible and wrong." A Mencken Chrestomathy (Vintage). $2^{\text {nd }}$ ed, 2008 retrieved from www.saveyourhands.com 